\title{
Residential security through the Home Assistant Platform
}

\author{
Laurentiu Munteanu ${ }^{1 *}$, Marius Cornel Suvar ${ }^{1}$, and Gheorghe Daniel Florea ${ }^{1}$ \\ ${ }^{1}$ National Institute for Research and Development in Mine Safety and Protection to Explosion \\ INSEMEX Petrosani, 32-34 General Vasile Milea Street, Petrosani, Romania
}

\begin{abstract}
The etymology of the word safety means: lack of danger; feeling of peace and confidence; shelter from any danger; feeling of security. A security system of goods, values and protection of people can be implemented through an automated platform, under the name of Home Assistant. This server software application can communicate with an easy control system for fast and two-way access, which can be mounted to a restricted main entrance. The automatic opening of the access door is controlled both by stand-alone RFID (Radio-Frequency Identification) modules, which act on an electric closing mechanism, and by an automation system configured in the Home Assistant application. The ultimate goal is the security of the premises through a system that can operate both locally and online via internet connection. The research methodology was based on the study of intelligent applications for residential access, described in the literature, aiming at integration into the open-source HA platform, by developing new code sequences. I come with this paper in support of those who want to bring an improvement in the existing automation system, respectively to encourage those who want an automated residence at a higher level.
\end{abstract}

\section{Introduction}

Information and technology are growing exponentially, in an accentuated rhythm. The technological environment creates opportunities to improve lives by modeling electronic devices with sensors.

Automation systems are found in more and more areas of everyday life. Also, even jobs are influenced by technological changes, namely automation / digitization [1]. By modernizing the technology there is an increase in productivity and quality of the final product.

There is a wide variety of smart devices on the market, such as: switches, sockets, bulbs, presence sensors, surveillance cameras, temperature, and humidity monitoring devices, etc. Almost any manufacturer of electronic equipment with WiFi connection also offers a customized application, with access and control from your mobile phone or computer. This is a disadvantage if you use more than one device from different manufacturers. In order not to be forced to use only equipment from the same

\footnotetext{
${ }^{*}$ Corresponding author: laurentiu.munteanu@,insemex.ro
} 
manufacturer, there is the choice of a specially built global program, which can combine and control all these existing equipment. This program is called Home Assistant.

The automation platform, Home Assistant, is based on the dynamic programming language, Python 3, through which you can track and control all the devices in the house.

"Home Assistant is an open-source home automation platform running on Python 3. Track and control all devices at home and automate control.’[2]

The programming language, Python, was created in 1989 by the Dutch programmer Guido van Rossum, who was one of the best software developers, who was given the title of "Benevolent dictator for life" (BDFL).

\subsection{Home Assistant application}

The Home Assistant software platform is a client-server service through which all the intelligent equipment in the residential network can be managed, controlled and monitored, even if this equipment comes from different manufacturers. The HA platform is a free, open-source global program to which advanced users and passionate automation developers from around the world have contributed, with the aim of combining all the intelligent equipment in the residence (building) [2].

To connect and create a suitable computer panel, electronic devices, regardless of the manufacturer, must contain sensors and have connectivity in the cable or WiFi network.

Depending on the devices chosen, there are a variety of possibilities and configuration options [3].

Regardless of the hardware system chosen for Home Assistant, it can be configured in two ways: the first is through a Docker container or console, an option recommended for experienced users, and the second way is to install the HASS.io operating system [4].

Like any beginner, when you install an operating system, you choose the simplest and safest option [5], HASS.io.

Smart electronic devices will communicate with the system through integrated tools called add-ons. These supplements are important for data transmission and reception, respectively for their combination and display mode. Currently there are over 1800 integrations provided by the community.

Immediately after installing the Home Assistant platform, you can customize the main interface or dashboard by choosing to install add-ons from the 2 major groups: official integrations and integrations offered by the home assistant community (fig. 1).

Most smart devices, installed on the local network, are automatically detected by HA and can be easily integrated.

The information and devices in the dashboard can trigger automation. One of the most important reasons for using HA is automation that responds immediately to things that happen. Any automation consists of a trigger and an action, optionally having one or more conditions [2]. As its name implies, the trigger is the event that triggers the automation rule.

The condition is used for specific cases of limitation of the automation rule. In other words, the action takes place only if the condition of the current state of some devices (closed / open) or other events are triggered or fulfilled. The action is the last stage of automation, which leads to the realization of an event, only if all the previous conditions are met. For example, the light (action) is turned on in the entrance hall, when the door is open (trigger), only in the time interval 06:00-12:00 PM (condition).

For beginners it is recommended to use blueprint automation (fig. 2), which contains ready-made automations by the community and can be easily attributed to general cases. 


\begin{tabular}{l|l} 
Home Assistant \\
Overview
\end{tabular} Mafficial add-ons

Fig. 1. Store add-ons

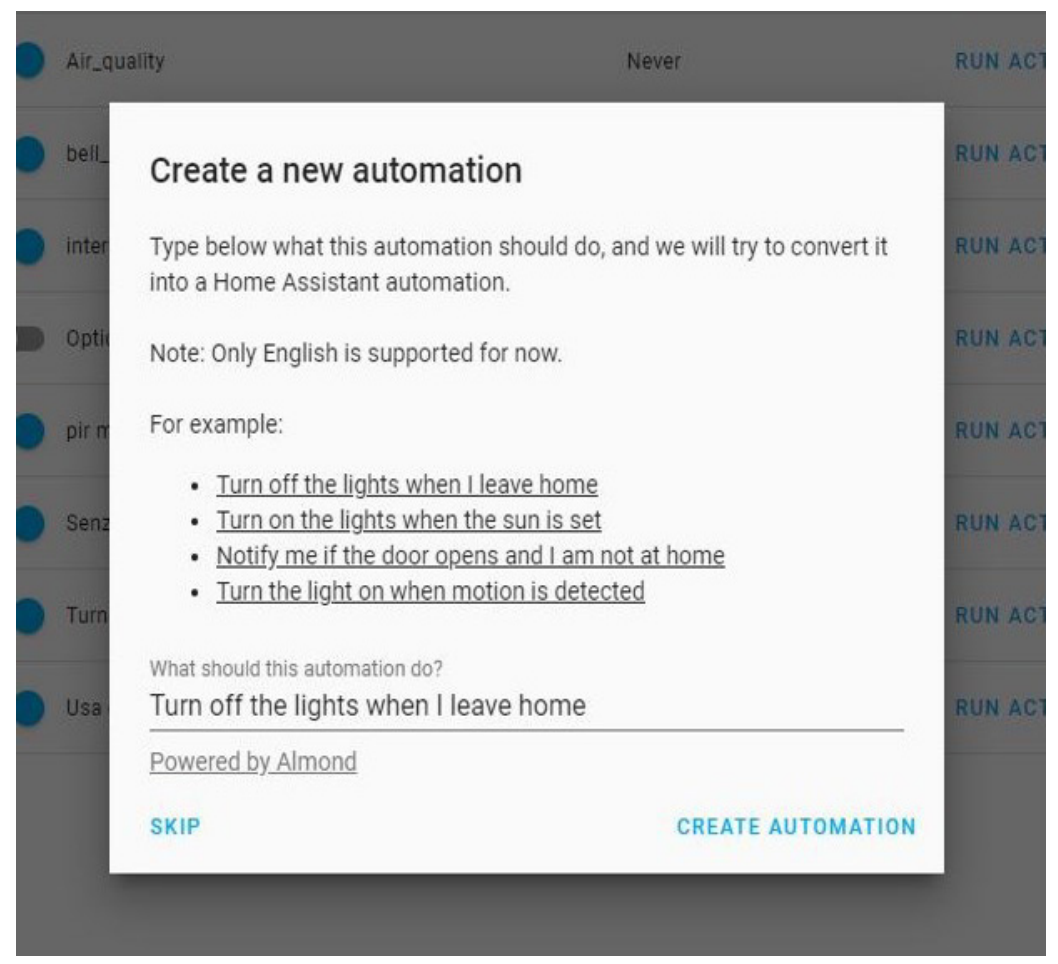

Fig. 2. Selection of blueprint automation 


\section{RFID technology}

\subsection{RFID system}

RFID is an advanced technological method, which is based on data recording and processing. Similar to barcode systems, the RFID reading system (fig. 3) consists of two types of devices: the RFID card reading mode, which also has the writing function, respectively RFID TAGs. A microcontroller-based computing system is used for data processing.

TAGs are objects of different shapes and sizes of the order of millimeters, with a small antenna, coil type and a memory chip for storing various information, including the unique ID code, fig. 4

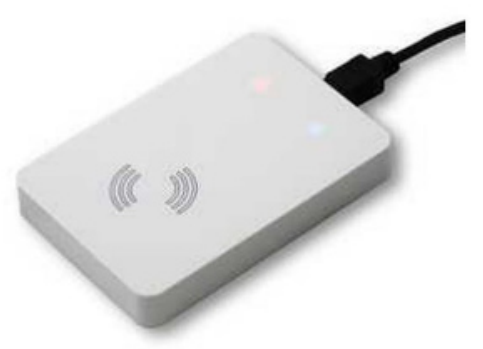

Fig. 3. RFID reader

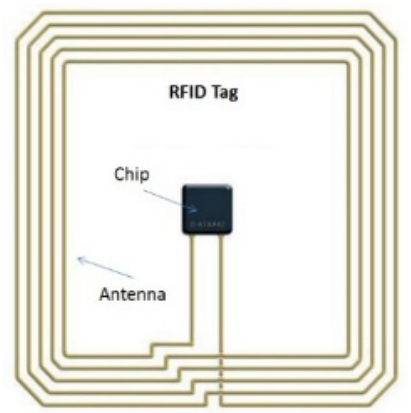

Fig. 4. The main components of a passive TAG

Compared to active TAGs, passive RFID TAGs (fig.5) have a long life and do not require an integrated power supply (battery). They are ideal in industrial environments where the dust and moisture content is high.

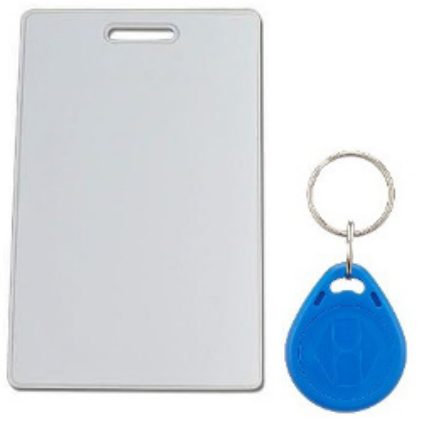

Fig. 5. Examples TAGs with unique code 


\subsection{Operation of an RFID system}

By approaching at a short distance, a card or special tags, the code is read using a low power radio frequency field.

The RFID system includes two major components: the RFID card reading mode and the mobile TAG, which are represented by a block diagram, fig. 6

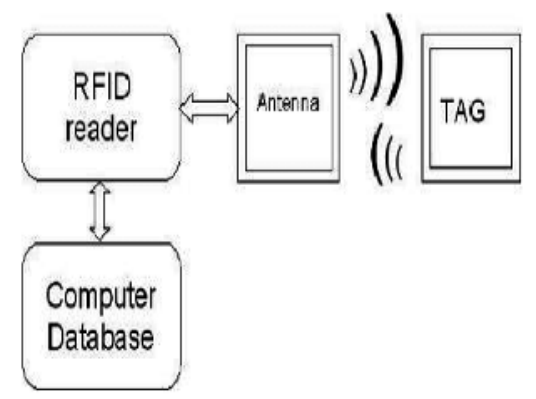

Fig. 6. Block diagram of the RFID system

In data transmission and reception, the reader and the mobile TAG have an internal antenna connected. At the reader, the antenna can be included in the housing or can be separated, located at a distance from the rest of the electronic components. To achieve high performance, the diameter of the reader's antenna must be larger than that of the TAG.

The reading module is related to the data processing system which can be a computer or more recently a dedicated microcontroller [6].

Electronic components of the reading module receive the signal from the proximity tag, and the microprocessor verifies and decodes the received data, with the possibility of recording [7].

The most important parameter in choosing the RFID system is the operating frequency. Depending on the reading distance, the frequency of the RFID module can be [8]:

- $125 \mathrm{kHz}$ (LF band - Low Frequency) up to $10 \mathrm{~cm}$

- 13.56 MHz (HF band - High Frequency) up to $1.5 \mathrm{~m}$

- $868 \mathrm{MHz}$ (UHF band - Ultra High Frequency) up to a few meters

One of the main advantages in using the RFID system in the industrial environment is the high communication speed and safety in operation.

\section{Automated security system through Home Assistant}

Any security system is composed of all the physical and technical elements called hardware components and the logical control part called the software component of the system. With the help of the hardware components used in the security system, the data is received, verified, recorded, processed and transmitted.

The main hardware module for intelligent closing is connected directly to the WIFI control module (fig. 7) via 4 connecting wires: 2 for $12 \mathrm{~V}$ power supply and 2 for output signal control.

The main module is equipped with a backup battery to power the internal circuit, necessary in case of an electrical outage from the mains. In this situation, the backup battery maintains the supply voltage for a sufficient period of time. After this time or if this battery is not installed, locking / unlocking can be done normally with a metal key.

The standard operating requirements for intelligent access control equipment are: voltage between $9-12 \mathrm{~V} \mathrm{DC}$, action current less than $350 \mathrm{~mA}$, working temperature 
between -40 and +70 degrees Celsius, working humidity up to $95 \%$, response time less than 0.5 seconds for RFID card unlocking, response time less than 0.3 seconds for RF remote control.

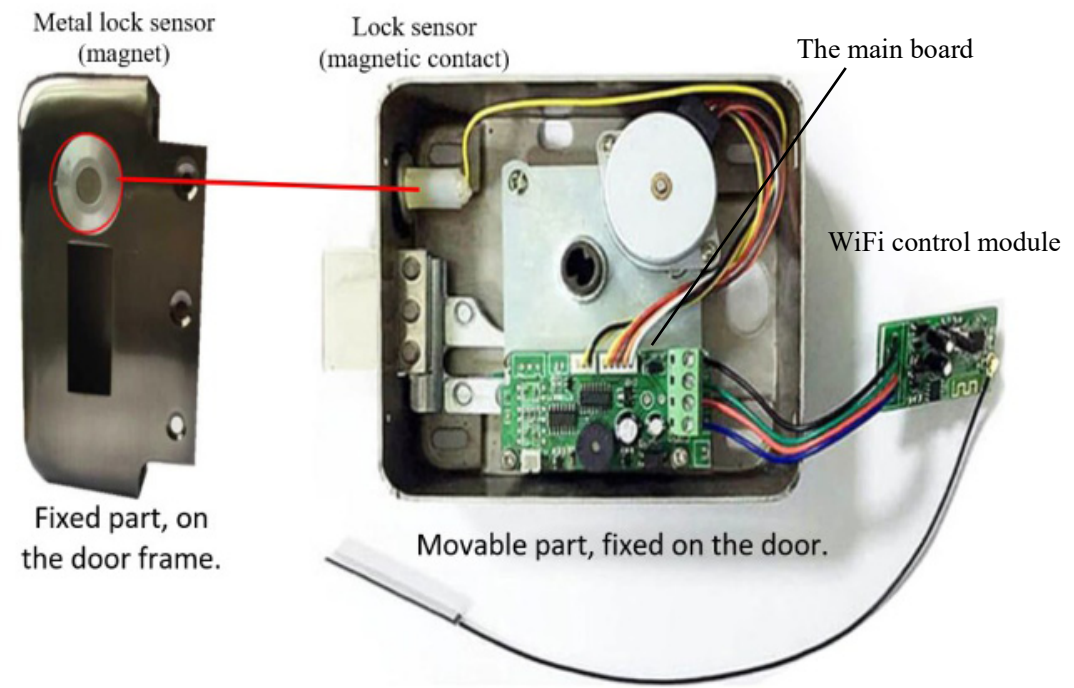

Fig. 7. Hardware components and magnetic contact

The security system is configured in HA, so that when the door is opened, a warning message is sent to the mobile phone. The alert is triggered by the closed / open state condition of the magnetic contact (fig.7). Thus, when the door is open, the magnetic sensor disengages the continuity of the "lock sensor" circuit and triggers the "open door" automation event.

In order to obtain the warning message and automation of the triggering of the "open door" event, the stages of the automation rule can be tested and verified according to fig.8.

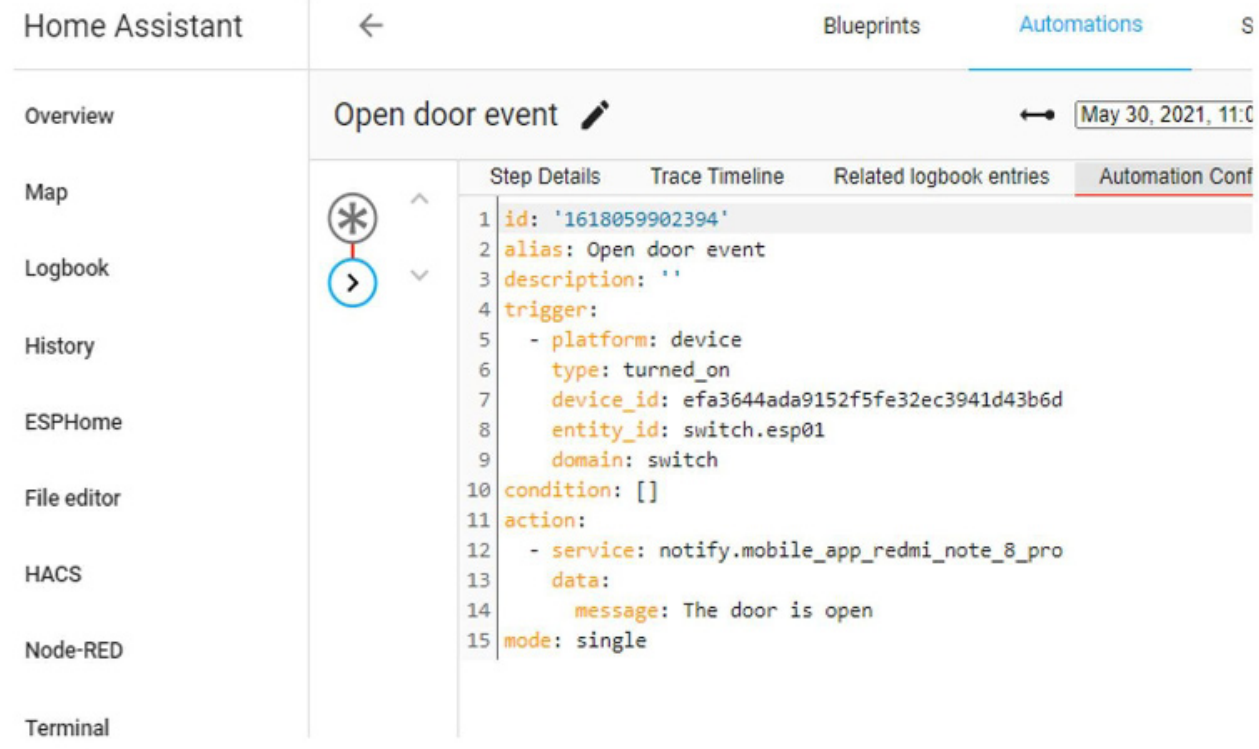

Fig. 8. Stages of the trigger automation event: "door open" 
In the case of the system presented in this paper, if the mobile phone is outside the internal network, the open door notification (fig10) is sent only if the HA platform is connected to the internet. In this case, the event notification is displayed on the mobile phone (fig.10).
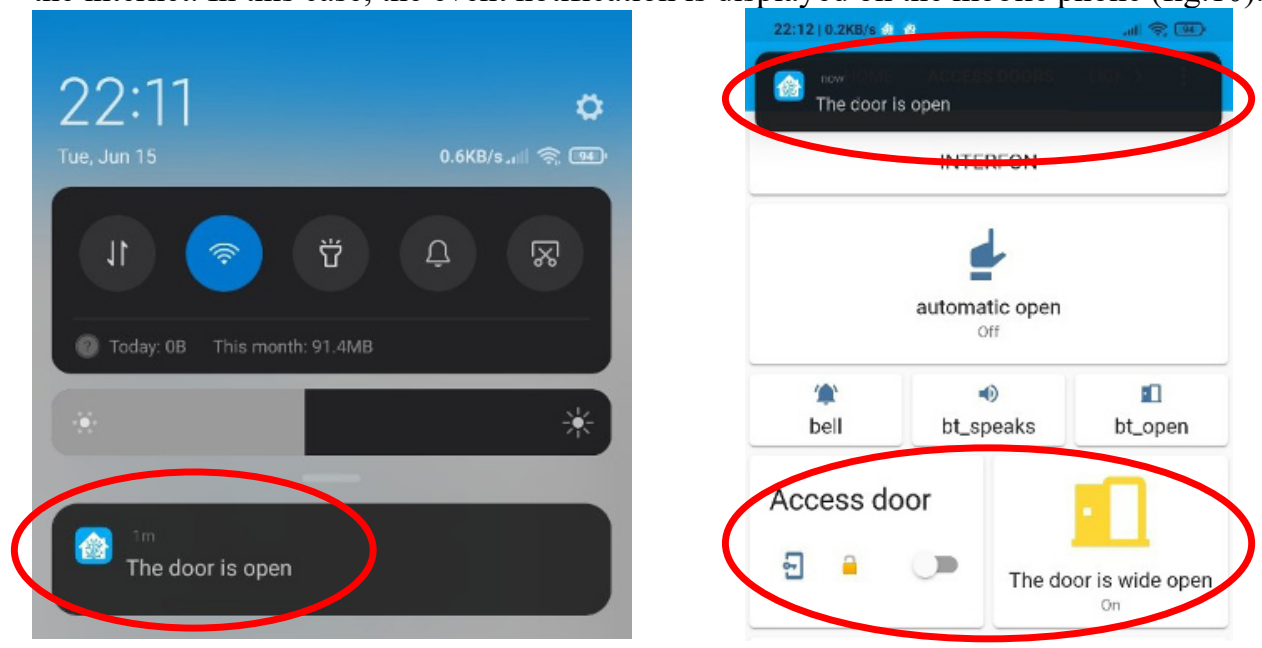

Fig. 10. Notification message on smartphone

In all cases, when a sensor changes its current state, a set of internal commands starts in the system, which triggers events. These events or conditioned states are recorded in descending order in the Logbook window (fig.13) and can be sent by warning or information messages to an email address or mobile phone.

The entire event history is recorded by the HA application and can be viewed in the History window (fig. 12).

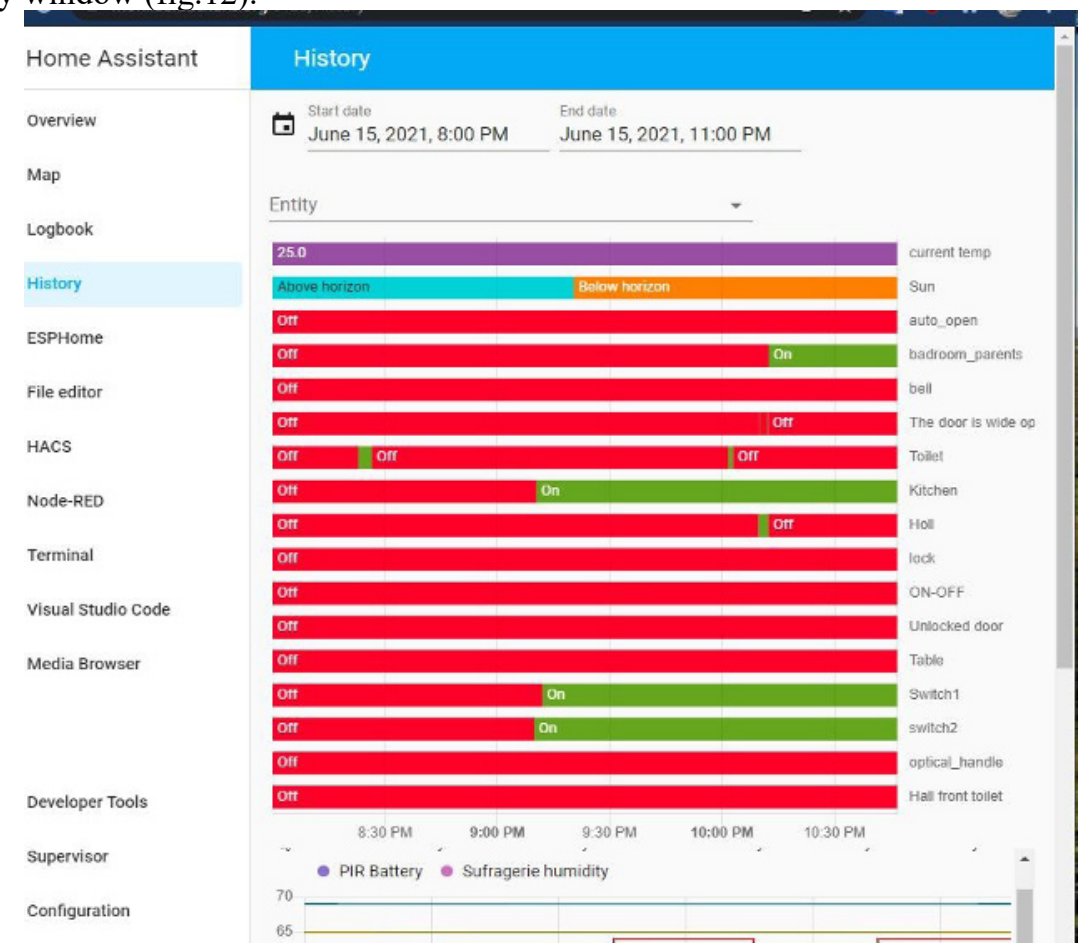

Fig. 12. Personalized history of HA events 
The use of the HA solution offers a number of advantages over third-party applications offered by automation equipment manufacturers. Among them we can mention:

- Centralization of smart IoT equipment from different manufacturers in a single platform

- Ensuring a high availability of services, even in case of temporary / accidental interruption of the internet connection

- Eliminating the vulnerability of a potential unauthorized access through the manufacturer's cloud service

- The HA platform allows the permanent development of automation solutions for residential / industrial use by adding additional programs to meet new facilities, created by members of the open source community or creating custom codes using programming languages supported by the platform (python, YAML, JSON, JavaScript, development tools based on visual programming, etc.)

\begin{tabular}{|c|c|c|}
\hline \multicolumn{3}{|c|}{$22: 11 \mid 1.5 \mathrm{~KB} / \mathrm{s}$ 을 } \\
\hline$\equiv$ & Logbook & C \\
\hline \multicolumn{2}{|c|}{$\begin{array}{l}\text { Start date } \\
\text { lune } 1520218.00 \text { PM }\end{array}$} & $\begin{array}{l}\text { End date } \\
\text { June 15, 2021, 11:00 PM }\end{array}$ \\
\hline Entity & & $\rightarrow$ \\
\hline \multicolumn{3}{|c|}{ June 15, 2021} \\
\hline 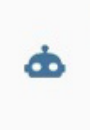 & \multicolumn{2}{|c|}{$\begin{array}{l}\text { Open door event has been triggered by state of } \\
\text { switch.esp01 } \\
\text { 10:09:53 PM - } 1 \text { minute ago - Show trace }\end{array}$} \\
\hline ๘ & \multicolumn{2}{|c|}{$\begin{array}{l}\text { Turn off light hall after } 30 \mathrm{~min} \text { has been } \\
\text { triggered by state of switch.kitchen2_intrare by } \\
\text { Holl } \\
\text { 10:09:43 PM - } 1 \text { minute ago - Show trace }\end{array}$} \\
\hline & \multicolumn{2}{|c|}{$\begin{array}{l}\text { Holl turned on } \\
\text { 10:09:43 PM - } 1 \text { minute ago }\end{array}$} \\
\hline & \multicolumn{2}{|c|}{$\begin{array}{l}\text { Toilet turned off } \\
10: 02: 58 \text { PM - } 8 \text { minutes ago }\end{array}$} \\
\hline & \multicolumn{2}{|c|}{$\begin{array}{l}\text { Toilet turned on } \\
\text { 10:01:42 PM - } 10 \text { minutes ago }\end{array}$} \\
\hline ৫. & \multicolumn{2}{|c|}{$\begin{array}{l}\text { Sun set } \\
9: 20: 25 \text { PM - } 51 \text { minutes ago }\end{array}$} \\
\hline 8 & \multicolumn{2}{|c|}{$\begin{array}{l}\text { Switch1 turned on } \\
\text { 9:12:01 PM - } 59 \text { minutes ago }\end{array}$} \\
\hline
\end{tabular}

Fig. 13. The latest events triggered in HA on the smartphone

The proposed code is generated by HA after completing the fields for the closed / open sensor status, fig.14 a) and b) 


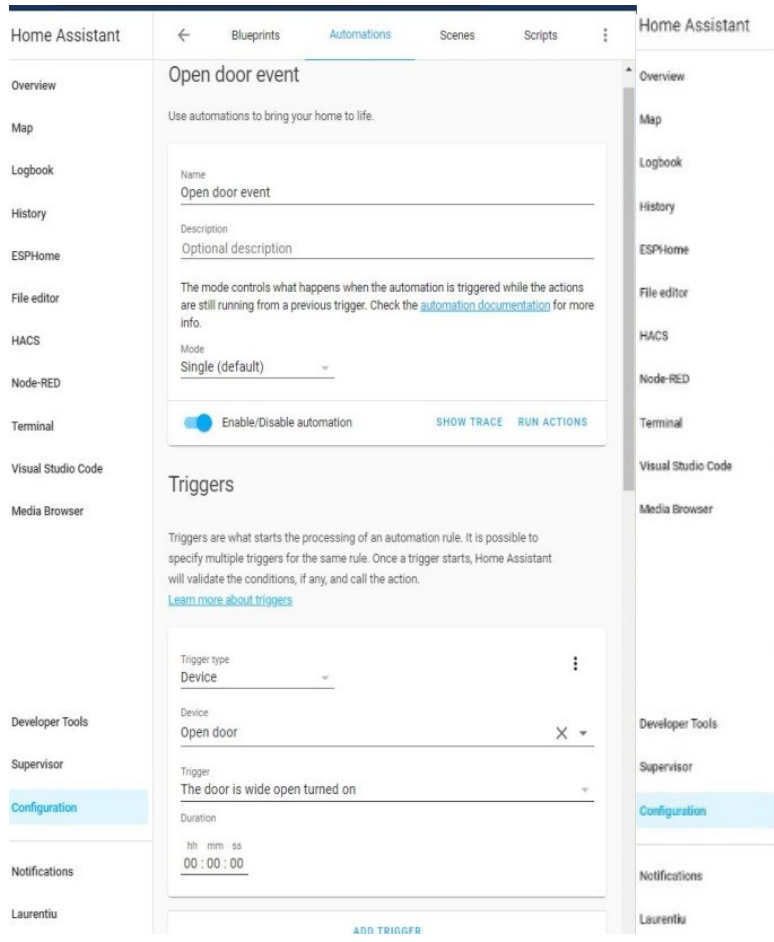

a)

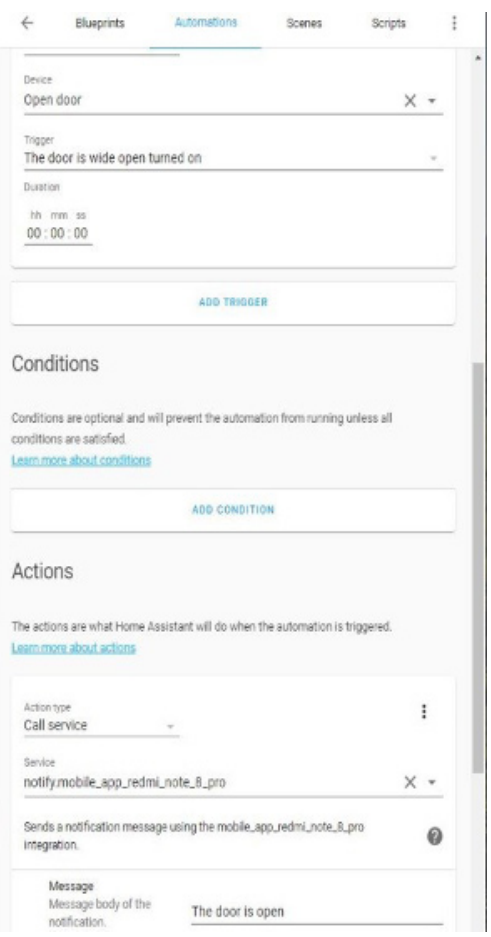

b)

Fig. 14. Open door event configuration

\section{Conclusions}

When you want to use a hub for smart device communication, you have to weigh very well its advantages and disadvantages and compare this hub with other global programs. If we choose one of the well-known hubs, such as Alexa or Google Home, the connection takes place exclusively in the online environment, where the information depends on:

- internet speed

- use of dedicated servers

- transmission of confidential data

- assuming a risk for the possibility of interception or espionage of data, up to the extreme case, control of your devices by unauthorized third parties.

Significant advantages of the intelligent security system:

- is addressed to owners in the residential sector

- it is easy to install and use

- has a high degree of security

- has a high communication speed and safety in operation

- has various unlocking methods: with key, RFID tag, remote control, through various applications for the phone or local network

- has the possibility to connect with other access control systems or intelligent video closing systems

- can communicate with intelligent systems in locations such as: home, offices, schools, hotels, factories, warehouses, institutions, restricted premises, etc.

A smart restricted access system combines, in addition to the electrical or electromagnetic mechanism, with various biometric or voice recognition modules, facial 
features, fingerprint scanner, RDIF tags, cards, access codes, etc. Always, the system must be chosen to minimize as much as possible the risk of a security breach, and for the highest degree of security, the system is combined with an alarm and surveillance.

HA allows creating, customizing, respectively automate your own preferences with electronic equipment, from the simplest by automatic control of a switch, to the most complex, which can take into account including time, weather, status of other sensors, etc. The configuration tools in the HA program are constantly being developed and updated by the community of passionate automation users in the open source environment.

Most smart devices have microcontrollers that can be reprogrammed by users. If the systems have been configured to communicate with predefined software, they can be removed from the manufacturer's cloud integration.

HA projects are the most sought after and recommended for automating smart devices used in various technological fields.

In the future, we want to modernize the access door unlocking system, automatically on the principle of "keyless entry" technology, similar to modern cars, when approaching at a distance of less than 1 meter.

\section{References}

1. https:/www.skills4future.eu/assets/resources/Getting-ready-for-the-future.pdf, [Accessed 10.05.2021]

2. https://www.home-assistant.io/docs/automation/basics, [Accessed 12.05.2021]

3. Home Assistant Team, Home Assistant Documentation, (Irvine, CA, 2021)

4. https://diyi0t.com/home-assistant-mqtt-tutorial, [Accessed 10.05.2021]

5. International Journal of Reliability, Quality and Safety Engineering, Shafiq Ul Rehman and Selvakumar Manickam, A Study of Smart Home Environment and it's Security Threats, Vol.23, 5(2016)

6. Revista Informatica Economica, Lixandroiu Radu, A Model of Traceability for Equipments Using RFID Technologies within an Integrated System, Vol.38, (2006)

7. AGIR Bulletin - Telecommunications, Pescaru G., Dragomir R., Puscoci S., Activities optimization using RFID technologies, no.2, (2008)

8. IOP Conference Series: Materials Science and Engineering, Thanapal P, Prabhu J and Mridula Jakhar, A survey on barcode RFID and NFC, 263 (2017) 